\title{
Las cadenas de televisión españolas en Internet: un estudio sobre la calidad de sus sitios web ${ }^{1}$
}

\author{
Jesús DÍAZ-CAMPO \\ Universidad Internacional de La Rioja (UNIR) \\ jesus.diaz@unir.net
}

Recibido: 2 de julio de 2013

Aceptado: 12 de febrero de 2014

\begin{abstract}
Resumen
Las cadenas de televisión se han visto obligadas a adaptar sus rutinas de trabajo a la denominada Sociedad Digital, que está cambiando las relaciones entre los medios de comunicación de masas y sus audiencias. Una de las consecuencias de estos cambios es que los usuarios quieren sentirse cada vez más protagonistas. El objetivo de este estudio es evaluar el grado de adaptación de las principales cadenas de televisión españolas a la Web 2.0. La metodología empleada está formada por 32 indicadores, agrupados entorno a siete parámetros que tratan de evaluar la calidad de sus sitios web y el uso que realizan de las herramientas para fomentar la interactividad. Los resultados muestran que el empleo de las herramientas propias de la web 2.0, el acceso a la información y el registro del usuario son los parámetros más presentes.
\end{abstract}

Palabras clave: Internet, televisión, interactividad, web 2.0

\section{Spanish television channels on Internet: a study on the quality of their websites}

\begin{abstract}
Television channels have been forced to adapt their work processes to the so called Digital Society, which is changing the relationship between mass media and their audiences. One of the consequences of these changes is that users want to feel increasingly participant. The aim of this study is to evaluate the degree of adaptation of the main Spanish TV channels to Web 2.0. The methodology we use consists of thirtytwo indicators, organized around seven parameters that try to evaluate the quality of their websites and their use of interactive tools. Results show that the use of the characteristics of Web 2.0; access to information and user registration are the most common parameters.
\end{abstract}

Keywords: Internet, television, interactivity, web 2.0

\section{Referencia normalizada}

DÍAZ-CAMPO, Jesús (2014): "Las cadenas de televisión españolas en Internet: un estudio sobre la calidad de sus sitios web". Estudios sobre el Mensaje Periodístico. Vol. 20, Núm. 1 (enero-junio), págs.: 67-83. Madrid, Servicio de Publicaciones de la Universidad Complutense.

Sumario: 1. Introducción. 2. Marco teórico. 3. Objetivos. 4. Metodología. 5. Resultados; 5.1. Interacción; 5.2. Publicación de contenidos; 5.3. Registro del usuario; 5.4. Acceso a la información; 5.5. Personalización del contenido; 5.6. Empleo de herramientas de la Web 2.0; 5.7. Presencia del medio en plataformas de la Web 2.0. 6. Conclusiones. 7. Referencias bibliográficas.

\section{Introducción}

Muy próximos a la celebración del vigésimo aniversario de la aparición en la web del primer ejemplar digital del Palo Alto Weekly o, lo que es lo mismo, el primer medio de comunicación publicado de manera regular en Internet, una recapitulación de lo su-

1 Trabajo parcialmente financiado por el Plan Propio de Investigación de la Universidad Internacional de La Rioja (UNIR). 
cedido desde entonces muestra, entre otras cosas, cómo los cibermedios de todo el mundo han ido poniendo en marcha diversas estrategias con desigual fortuna pero con idéntico objetivo: tratar de explotar al máximo las posibilidades que les ofrecía el nuevo soporte.

En esa evolución no han faltado, en especial en los primeros momentos, quienes han pronosticado el fin de los medios de comunicación tradicionales, una tendencia inicial que más tarde se ha transformado en una visión radicalmente diferente: la de Internet como aliado de los soportes tradicionales e instrumento ideal para la explotación y promoción de contenidos.

Una de las vías más elementales para intentar alcanzar ese objetivo es aumentar la calidad de los sitios web, a través de la implementación constante de nuevas funcionalidades y buscando, entre otras cosas, una mayor interacción con el usuario. Pues bien, este estudio trata de determinar cuál es el uso que están haciendo las principales cadenas de televisión españolas de Internet y para ello se analizan los sitios web de las cadenas que gozan de mayores índices de audiencia de acuerdo a los datos más recientes del Estudio General de Medios (EGM) en el momento de la realización del estudio.

\section{Marco teórico}

En contra de lo que vaticinaron numerosos autores, como Pérez Silva (2000), la televisión no ha muerto, aunque sí que se ha visto sometida a cambios muy profundos, un proceso en el que algunas de las claves que lo definen son las que señaló el mismo autor, en especial el hecho de buscar un mayor acercamiento a la audiencia, ofreciendo una misma televisión para todos pero que al tiempo suponga una experiencia única para cada telespectador (Pérez Silva, 2000: 68).

Por el contrario, los medios de comunicación en general, y las cadenas de televisión en particular, han tratado de sacarle el máximo rendimiento a las características del nuevo medio, Internet, una herramienta con unas capacidades que aumenta exponencialmente las posibilidades en términos de audiencias, generación de contenidos y también facturación. Por eso, si hubiese que elegir un concepto que definiera cuál ha sido el devenir de las cadenas de televisión en todo este tiempo, sin duda sería el de convergencia, entendida tal y como la definen Salaverría, García-Avilés y Masip (2010: 48):

"un proceso multidimensional que, facilitado por la implantación generalizada de las tecnologías digitales de telecomunicación, afecta al ámbito tecnológico, empresarial, profesional y editorial de los medios de comunicación, propiciando una integración de herramientas, espacios, métodos de trabajo y lenguajes anteriormente disgregados, de forma que los periodistas elaboran contenidos que se distribuyen desde múltiples plataformas, mediante los lenguajes propios de cada una".

Esa convergencia se ha dado en múltiples ámbitos, tanto estructurales como en los procesos de producción y difusión de contenidos (Tremayne, Weiss y Alves, 2007; Paterson y Domingo, 2008, Navarro, 2009), y ha afectado a las relaciones del medio con su audiencia, con la publicidad y con los propios profesionales. Y es que, en palabras de López García, 2011: 91), el cambio es tan profundo que lo único que per- 
manece es "lo básico, la esencia del periodismo -"el periodismo puro y duro"-, y la necesidad de los ciudadanos de disponer de información para poder moverse en la sociedad". Se trata de un nuevo escenario cuyas principales características, que han sido suficientemente definidas por varios autores, se refieren a la multimedialidad, la hipertextualidad, la personalización, la interactividad, la inmediatez o la posibilidad de actualización permanente, entre otras (Cabrera González, 2000; Orihuela, 2002; Cebrián, 2009; Masip, Díaz-Noci, Domingo, Mico-Sanz y Salaverría, 2010).

Al mismo tiempo, las cadenas han tenido que dar respuesta a una doble cuestión: los cambios que ha ido experimentando la propia naturaleza de la red, y las nuevas demandas de los usuarios. En el caso de la televisión, ambas han venido marcadas por un acontecimiento del que se puede decir que ha establecido un antes y un después: la aparición de Youtube en 2005, que acrecentó enormemente el interés de los usuarios por ver vídeos a través de la pantalla del ordenador, algo que con el tiempo obligó a las cadenas -reticentes en un principio ante el peligro de perder audiencia- a volcar sus contenidos en la web tratando, eso sí, de buscar la interrelación entre ambos soportes.

La entrada en escena de Youtube, unida al surgimiento de la llamada web 2.0, supone el germen de otra de las iniciativas más importantes en términos de interactividad y de personalización: los servicios a la carta, esto es, el hecho de que el usuario pueda adaptar y hasta diseñar la programación en función de sus propias preferencias personales.

El último paso en esa evolución sería el nuevo papel de los usuarios como autores o, lo que es lo mismo, el periodismo participativo en sus diferentes grados de elaboración (Díaz-Noci, 2010), con una importante novedad, los usuarios participan en todas las fases del proceso de producción de la información, recogida, análisis y difusión (Bowman y Willis, 2003), en un proceso favorecido precisamente por las propias características de los cibermedios, como la multimedialidad, la accesibilidad o la retórica hipertextual, elementos todos ellos que tienen en común precisamente el hecho de facilitar la participación de la audiencia en el proceso comunicativo (Cabrera González, 2005).

De todos modos, la necesidad de adaptación constante a los cambios permanentes no ha ayudado precisamente a marcar una estrategia clara y continuada en el tiempo y ha provocado que siga sin existir un modelo claro para lograr esa participación (Lara, 2005). De ahí que las dudas y la experimentación tampoco hayan faltado en una trayectoria en la que tanto los modelos de negocio como los procesos de producción de contenidos han ido variando una y otra vez.

En el caso de España, una primera fuente de interés es el estudio Televisión: Tradicional vs. Online, elaborado por la Asociación para la Investigación de Medios de Comunicación (AIMC) y presentado en 2012, algunas de cuyas principales conclusiones eran las siguientes:

1. Un $18 \%$ de los internautas accede en el mismo día a la televisión tradicional y a la televisión a través de Internet. La cifra aumenta hasta el 54\% hablando del último mes.

2. El 11\% del consumo de televisión entre los internautas se realiza a través de Internet. 
3. La mayoría de los que acceden a los dos tipos de televisión afirman de manera mayoritaria, $72 \%$, que ven la televisión convencional el mismo tiempo que antes, de manera que su consumo total de televisión se ha incrementado.

4. El 88\% de los que sólo ven televisión a través de Internet no tienen televisor en casa.

En cuanto a los principales estudios llevados a cabo hasta la fecha, destaca el de Meso, Palomo y Ganzábal (2009), quienes analizaron la presencia de elementos propios del periodismo participativo en seis cadenas, Rtve.es; Antena3.com, Telecinco.es, Plus.es, LaSexta.com y Cuatro.com. Una de las conclusiones más relevantes de este análisis es que las cadenas nacionales españolas suponían en ese momento una especie de laboratorio de pruebas. Asimismo, los autores destacaban que a finales de 2008 las seis cadenas analizadas ponían a disposición del usuario un completo de opciones de participación tras haber incorporado diversas mejoras a sus páginas web con el objetivo de mejorar su usabilidad y dinamismo y de hacerlas más participativas.

Por lo que respecta a otras investigaciones, un trabajo de Meso y Larrondo (2011), documenta varias de ellas, como la de Pérez y Santos (2009) sobre televisiones locales del País Vasco, o la de García de Torres y Pou (2000) sobre televisiones locales de Valencia. A ellas habría que sumarles trabajos como los de Arjona (2009), centrado en la página web de RTVE; el de Lerma (2011) también referido sobre las televisiones locales de Valencia; el de García Torre (2012), quien analiza el profundidad la página web de TVG, o el de Odriozola (2012), que examina las portadas de los cibermedios generalistas, entre ellos varias televisiones. Pues bien, prácticamente todos estos trabajos coinciden en una idea, ya sea una televisión pública o privada, local autonómica o estatal, todas ellas tienen la obligación hoy por hoy de estar presentes en Internet y de ofrecer además las máximas posibilidades a sus usuarios. Asimismo, varios de ellos señalan la conveniencia de hacer que la audiencia se sienta protagonista como paso necesario para fidelizarla. En todo caso, el trabajo de Meso y Larrondo, referido a televisiones autonómicas, reconoce que "son pocos los estudios que cubren en el panorama español este análisis del periodismo participativo en televisión debido a su reciente aparición".

De ahí la necesidad de realizar un análisis con carácter más general para comprobar si las principales cadenas de televisión españolas han realizado a día de hoy una adaptación adecuada al entorno de la web.

\section{Objetivos}

Tomando como punto de partida el marco teórico establecido en el epígrafe anterior, la presente investigación se marca los siguientes objetivos:

1. Analizar si las principales cadenas de televisión han realizado a día de hoy una adaptación adecuada al entorno de Internet y determinar cuál es el nivel de calidad de sus respectivos sitios web.

2. Establecer cuáles son los aspectos o parámetros que reflejan unos niveles más altos de adaptación y calidad y qué otros indicadores son susceptibles de mejora. 
3. Comprobar si existe algún tipo de relación directa entre el índice de audiencia de una cadena de televisión y el nivel de calidad de su sitio web. En este sentido, se trata de determinar también si existen diferencias significativas entre las cadenas en función de su ámbito de actuación (públicas/privadas; estatales/autonómicas).

Para ello se ha analizado una muestra conformada por las diez cadenas con mayor número de espectadores según los datos del Estudio General de Medios acumulado de las tres oleadas de 2012. A partir de esa lista, se ha considerado oportuno suprimir la división entre La 1 y La 2 (ambas aparecían entre las diez primeras cadenas) y evaluar únicamente el sitio web de RTVE, por cuanto del análisis realizado se ha comprobado que las funcionalidades de uno y otro son idénticas, además de que el sitio web matriz de ambos es el mismo. De este modo, se han evaluado las siguientes cadenas: Tele 5 (telecinco.es), Radio Televisión Española (rtve.es), Antena 3 (antena3.com), Cuatro (cuatro.com), La Sexta (lasexta.com), Canal Sur (canalsur.es), TV3 (tv3.cat), Canal 9 (rtvv.es), Telemadrid (telemadrid.es), y Televisión de Galicia (crtvg.es).

\section{Metodología}

Para evaluar el grado de interactividad de los sitios web de las diez cadenas analizadas se ha utilizado un método que se considera plenamente contrastado y cuyo origen se encuentra en el campo de la Documentación: se trata de una propuesta del profesor Lluís Codina, recogido en diversos textos ${ }^{2}$ y una de cuyas últimas versiones se ha publicado recientemente (Rodríguez-Martínez, Codina y Pedraza-Jiménez, 2012).

Se trata de una herramienta que hace especial énfasis en la interacción y, más en particular, en la personalización, pero sin dejar de lado otros aspectos relacionados con la usabilidad y la arquitectura de la información (Rodríguez-Martínez, Codina y Pedraza-Jiménez, 2012: 62). Por lo demás, en su elaboración ha tenido en cuenta otros trabajos y modelos de análisis (Zamith, 2008; Guallar y Abadal, 2009; Palacios y Díaz-Noci, 2009; Rodríguez-Martínez, Codina y Pedraza-Jiménez, 2010).

En concreto, este método de evaluación, surgido del análisis de una multitud de sitios web entre los que se encontraban los de diarios internacionales y nacionales, canales de televisión, blogs elaborados por periodistas y sitios web de periodismo ciudadano, entre otros Rodríguez-Martínez, Codina y Pedraza-Jiménez, 2012: 63) se basa en el establecimiento de dimensiones, parámetros e indicadores que permitan examinar sitios web de medios de comunicación y conocer si han hecho o no una adaptación adecuada al entorno de la web (Díaz-Noci, 2009).

El modelo de análisis contempla seis dimensiones: cooperación, participación, creación de contenido, acceso al contenido, socialización y comunicación; mientras que emplea ocho parámetros: interacción usuario-medio de comunicación; publicación de contenidos creados por los usuarios; registro del usuario; acceso a la información; canal de contenido personalizado; el medio ofrece distintas versiones de su informa-

2 Muchos de esos textos pueden consultarse en la página web www.lluiscodina.com. 
ción; empleo de herramientas de la Web 2.0; y plataformas de la Web 2.0 en las que tiene presencia el medio de comunicación.

A su vez, cada uno de estos parámetros agrupa varios indicadores, que suman un total de 36:

1. Parámetro 1: interacción medio de comunicación-usuario.

1.1. Comunicación con el autor de la noticia.

1.2. Contacto con la redacción del medio de comunicación.

1.3. Comentar noticias publicadas por el medio de comunicación.

1.4. Votación de noticias publicadas por el medio de comunicación.

1.5. Comentar entradas publicadas en los blogs del medio de comunicación.

1.6. El usuario puede modificar o corregir contenido publicado por el medio.

2. Parámetro 2: publicación de contenidos creados por los usuarios.

2.1. Creación de blogs por los usuarios.

2.2. Publicación de textos escritos por los usuarios.

2.3. Publicación de fotos tomadas por los usuarios.

2.4. Publicación de vídeos realizados por los usuarios.

2.5. Sección exclusiva para contenido creado por los usuarios.

3. Parámetro 3: registro del usuario.

3.1. Registro por parte del usuario en el medio.

3.2. Contacto con otros usuarios registrados.

4. Parámetro 4: acceso a la información.

4.1. Acceso a la información a través de la portada.

4.2. Acceso a la información a través de secciones.

4.3. Acceso a la información a través de noticias relacionadas.

4.4. Acceso a la información a través del buscador.

4.5. Acceso a la información a través del mapa web.

4.6. Acceso a la información a través de la recomendación de los usuarios.

4.7. Acceso a la información a través de plataformas externas de la Web 2.0.

5. Parámetro 5: personalización de la información.

5.1. Adaptación de la interfaz del sitio web del medio de comunicación en función de los contenidos de interés para el usuario.

5.2. Sindicación de contenidos del medio de comunicación a través del móvil o correo electrónico.

5.3. Suscripción de alertas o boletín electrónico.

6. Parámetro 6: el medio ofrece distintas versiones de su información.

6.1. Versión impresa del medio.

6.2. Versión global.

6.3. Versión actualizada de forma constante.

6.4. Versión impresa adaptada a la Web 2.0.

7. Parámetro 7: empleo de herramientas de la Web 2.0.

7.1. Compartir información con otros usuarios.

7.2. Blogs vinculados al medio de comunicación.

8. Parámetro 8: plataformas de la Web 2.0 en las que tiene presencia el medio de comunicación.

8.1. Presencia del medio de comunicación en plataformas audiovisuales.

8.2. Presencia del medio de comunicación en plataformas de imágenes.

8.3. Empleo de redes sociales propias.

8.4. Presencia del medio de comunicación en redes sociales profesionales externas. 
8.5. Presencia del medio de comunicación en redes sociales de amistad.

8.6. Presencia del medio de comunicación en plataformas de microblogging.

8.7. Vinculación entre el sitio web del medio de comunicación y las plataformas sociales.

En esta investigación se decidió omitir el parámetro número 6 así como sus correspondientes indicadores, por considerarlos, a diferencia del resto, poco apropiados para los medios audiovisuales y limitados al análisis de medios impresos. De este modo se analizaron un total de 32 indicadores agrupados bajo siete parámetros generales.

El análisis de las páginas web se realizó a lo largo de un mes, durante los días 8 de enero a 8 de febrero, observando hasta en cuatro ocasiones diferentes la presencia o no de los indicadores señalados.

A diferencia del método original que proponía en algunos indicadores concretos una puntuación de 0 a 3 (Rodríguez-Martínez, Codina y Pedraza-Jiménez, 2012), se ha considerado más objetivo que cada pregunta sólo pueda ser puntuada con un $0 \mathrm{o}$ un 1, respondiendo a la ausencia o presencia de cada característica respectivamente y añadiendo una puntuación general para medir el grado de interactividad.

\section{Resultados}

\subsection{Interacción}

La interacción medio de comunicación-usuario hace referencia al establecimiento de una relación comunicativa entre uno y otro, en la que ambos interactúan, lo que supone un cambio radical en el proceso de construcción del discurso periodístico, puesto que el público adquiere un gran protagonismo, que se traduce en su participación en ese proceso, algo que hasta ahora se había dado muy escasamente, a través de mecanismos como las cartas al director o las llamadas telefónicas a los programas de radio y televisión, por poner dos ejemplos. En ese sentido, las características de Internet como medio de comunicación posibilitan que ese protagonismo sea mucho mayor y que el usuario pueda incluso ejercer el rol de emisor.

Pues bien, los resultados en este apartado (Tabla 1) muestran en primer lugar que ninguna de las cadenas analizadas cumple todos los indicadores, y que es Telemadrid la que reúne un mayor número de ellos debido, principalmente, a que es la única de las que permite al usuario ponerse en contacto, a través del correo electrónico, con el autor de la noticia. Sin embargo, la opción de contactar con la redacción o sección correspondiente mediante la misma vía sí que es ofrecida de forma mayoritaria.

Del mismo modo, las cadenas apuestan mucho más decididamente por ofrecer la posibilidad de comentar la noticia que por permitir que el usuario califique la información en función de que le haya generado más o menos interés. Así, siete de las 11 cadenas analizadas ofrecen la primera opción, mientras que únicamente dos contemplan la segunda. En este sentido, hay un matiz importante relacionado con esta disyuntiva: el usuario que vota una noticia en canalsur.es o en crtvg.es no necesita registrarse para ello, mientras que el usuario que pretenda comentar una información en antena3.com, lasexta.com, tv3.cat, telemadrid.es y crtvg.es sí que tendrán que hacerlo necesariamente. Es curioso el caso de la cadena autonómica gallega, que sí exige este requisito para el comentario pero no para la valoración de la noticia. 
Por otro lado, telecinco.es y cuatro.com ofrecen ambas posibilidades a la hora de comentar la noticia: el usuario puede optar por registrarse previamente o puede valorar la información de manera anónima. Por su parte, rtve.es no ha activado la opción de comentar las noticias - ni siquiera registrándose es posible- pero sí que la ofrece en el caso de los espacios específicos de la mayoría de sus programas y series.

En la misma línea, la opción de permitir los comentarios en las entradas de los blogs también es mayoritaria, ya que las únicas dos cadenas que no permiten esta posibilidad, lasexta.com y crtvg.es no publican blogs en sus respectivos sitios web.

Tabla 1: Interacción medio de comunicación-usuario. Fuente: Elaboración propia

\begin{tabular}{|c|c|c|c|c|c|c|c|c|c|c|}
\hline Indicador & (5) & rtve & $\theta$ & $\infty$ & (b) & 米 & 3 & etviv & 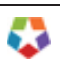 & c \\
\hline Comunicar autor & & & & & & & & & $x$ & \\
\hline Contactar redacción & $x$ & $x$ & $\mathrm{x}$ & $\mathrm{x}$ & & $x$ & $\mathrm{x}$ & $x$ & $x$ & $\mathrm{x}$ \\
\hline Comentar noticias & $\mathrm{x}$ & & $x$ & $\mathrm{x}$ & $x$ & & $x$ & & $x$ & $x$ \\
\hline Votar noticias & & & & & & $x$ & & & & $\mathrm{x}$ \\
\hline Comentar blogs & $x$ & $x$ & $x$ & $\mathrm{x}$ & & $\mathrm{x}$ & $x$ & & $x$ & \\
\hline Modificar contenido & & & & & & & & & & \\
\hline
\end{tabular}

\subsection{Publicación de contenidos}

Aspecto íntimamente relacionado con el examinado en el epígrafe anterior, supone dar un paso más en la búsqueda del protagonismo para las audiencias que, no sólo participan en el proceso comunicativo -a través de herramientas como foros, comentarios, encuestas, chats, blogs o videoconferencias- sino que producen sus propios contenidos, principalmente en forma de textos, fotografías o vídeos.

Este tipo de iniciativas puede interpretarse, por otro lado, como una reacción frente al auge de plataformas como las ya citadas de Youtube u otras similares como Flickr, que permiten igualmente que el usuario desempeñe el rol de emisor, con lo que se constituyen en una especie de nueva competencia (Cabrera González, 2009: 168) para las empresas de comunicación, que deciden por ello otorgar un protagonismo inusitado al público en un proceso que, por otra parte, no sólo fomenta la relación medio de comunicación-audiencia, sino también las relaciones entre los propios usuarios.

Pues bien, los resultados obtenidos muestran claramente que el nivel de cumplimiento de los indicadores correspondientes al Parámetro 2 (publicación de contenidos) es mucho menor que el del Parámetro 1 (interacción con el usuario) y ponen en entredicho esa tendencia hacia la conversión del usuario en autor de la que se habló antes, ya que únicamente telemadrid.es y, en menor medida, cuatro.com y rtvv.es, ofrecen algunas oportunidades al público en este sentido, mientras que las siete cadenas restantes no tienen activo ninguno de los indicadores. La cadena autonómica madrileña aparece como la más activa en este sentido al ofrecer al usuario la posibilidad de publicar todo tipo de contenidos -textos, fotos y vídeos- creados y/o elaborados por él mismo, mientras que cuatro.com y rtvv.es permiten la publicación de fotos y vídeos, pero no la de textos.

Por otro lado, el estilo de estas secciones suele destacar por ser bastante desenfadado y parece dirigido principalmente al público juvenil, a juzgar por sus contenidos 
y por su estética, como puede verse en la Figura 1, que muestra la portada de Vive Cuatro, la sección de participación en el sitio web de cuatro.com.

Figura 1: Portada de Vive Cuatro, sección de participación de cuatro.com.

Fuente: cuatro.com (31-01-2013)

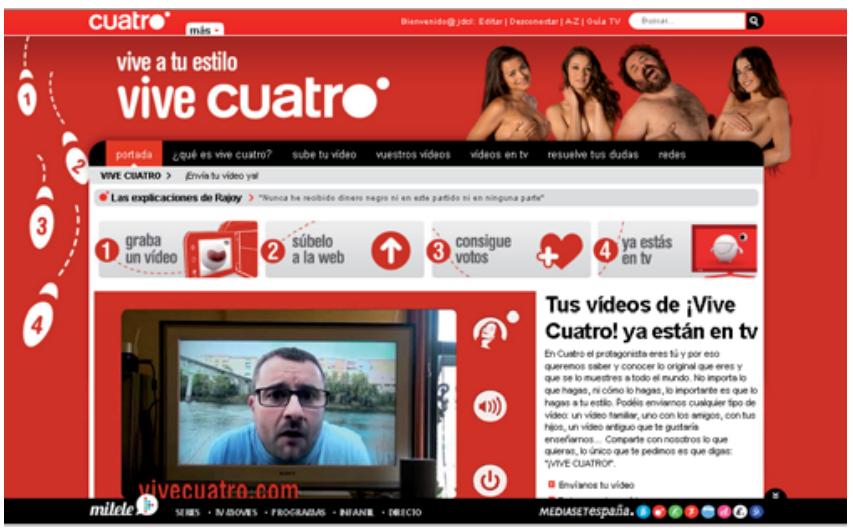

En el caso de telecinco.es, este tipo de iniciativas sí se ha promovido con ocasión de la celebración de eventos concretos, que han tenido gran repercusión en términos de audiencia para la cadena. Uno de los más recientes fue la celebración de la Eurocopa de fútbol el pasado verano, durante la cual se invitaba a los usuarios a enviar sus vídeos animando a La Roja, nombre por el que se conoce a la selección española de fútbol.

En este sentido, hay que reseñar que el mencionado estudio de Meso, Palomo y Ganzábal, fechado en 2009, recogía dos iniciativas de este tipo y de reciente implantación por aquel entonces: los espacios Yo, cámara, de telecinco.es y la creación del espacio Comunidad en lasexta.com. Ni uno ni otro parecen seguir activos o, al menos, eso es lo que se desprende del análisis de los respectivos sitios web.

Es significativo también el hecho de que ninguna de las cadenas permite la creación de blogs y que igualmente tampoco ninguna de ellas ha puesto en marcha una sección dedicada en exclusiva a la publicación de contenido generado por los usuarios, algo que sí se da, por ejemplo, en ciertos medios impresos ${ }^{3}$.

\subsection{Registro del usuario}

El registro previo es un requisito que la mayoría de los medios de comunicación exige al usuario para que éste pueda utilizar de manera activa las diversas herramientas interactivas: comentar las noticias o los blogs, recomendar algún contenido, enviar textos, fotografías o vídeos para su publicación, etc. En un segundo nivel, este registro suele ser también necesario para poder relacionarse con otros usuarios, ya sea a través de foros o de comunidades virtuales que se crean en el sitio web para que unos y otros puedan interactuar entre sí.

3 El trabajo en el que se basa la metodología de análisis de esta investigación cita el caso del diario lavanguardia.es, Rodríguez Martínez, Ruth; Codina, Lluís y Pedraza Jiménez, Rafael, op. cit., pp. 72-73. 
En el caso de las cadenas de televisión analizadas, la posibilidad de registrarse como usuario está presente de manera prácticamente unánime, ya que todas ellas la contemplan salvo canalsur.es. También es mayoritaria la posibilidad de contactar con otros usuarios, presente en los sitios web de Telecinco, RTVE, Antena 3, Cuatro, La Sexta, TV3 y Canal 9, pero no en Canal Sur, Telemadrid y TVG. No obstante, como ya se ha comentado y como se deduce también de los datos del resto del análisis, la variedad de opciones que implica el registro varía de unas cadenas a otras, e incluye en muchos casos un aspecto no recogido en la ficha de análisis utilizado: la participación en concursos u otro tipo de eventos promocionales.

\subsection{Acceso a la información}

La ingente cantidad de información que puede encontrarse en el sitio web de cualquiera de las cadenas analizadas convierte en imprescindible la labor de gestión y clasificación de la misma así como el hecho de facilitar al máximo la búsqueda de esa información por parte de los usuarios. Por ello, este tipo de herramientas pueden no ser las más complejas desde el punto de vista de la configuración pero su importancia es máxima a la hora de orientar al público.

Pues bien, este parámetro es el que ha registrado un mayor grado de cumplimiento en el análisis, con presencia unánime en cuatro de los siete indicadores analizados: en el acceso por portada, por secciones, y a través del buscador y de Facebook.

Desde el punto de vista de los contenidos, es igualmente interesante el acceso a través de noticias relacionadas, es decir, cuando consulta una determinada información encuentra enlaces a otros contenidos de temática similar que pueden servir para contextualizar la información original. Se trata de un recurso ofrecido por todas cadenas analizadas salvo tv3.cat y rtvv.es, las cuales tampoco cuentan con acceso a la información a través de la recomendación de los usuarios. Este último recurso tampoco está presente en la página web crtvg.es.

Del mismo modo, es reseñable el hecho de que la mitad de cadenas, rtve.es, antena3.com, canalsur.es, tv3.cat y telemadrid.es cuenta con mapa web, un recurso muy útil por constituir un índice de las distintas páginas del sitio a las que pueden acceder los usuarios.

Por último, un análisis de este parámetro por cadenas revela una presencia algo mayor de los indicadores en las cadenas de ámbito estatal que en las autonómicas, si bien hay dos de éstas últimas, canalsur.es y telemadrid.es, que incluyen todos los tipos de acceso analizado. Por el contrario, tv3.cat, rtvv.es y rtvg.es son las que muestran mayores carencias, si bien en todas ellas están presentes más de la mitad de indicadores analizados.

\subsection{Personalización del contenido}

Una de las máximas expresiones del acceso a la información de forma personalizada es probablemente el indicador 5.1 del modelo de análisis empleado: la adaptación de la interfaz del sitio web de la cadena en función de los intereses del usuario, esto es, que el medio comunicación permite al usuario seleccionar la información que le interesa e incluso rediseñar con ella el sitio web. Asimismo, en algunas ocasiones el usuario, previo registro, puede llegar a acceder directamente a su página personalizada cada vez que vi- 
sita el sitio web. Se trata de una opción que sí ofrecen algunos medios de comunicación internacionales como la BBC, pero que no se ha encontrado en los sitios web de ninguno de las cadenas analizadas, las cuales sí que han activado de manera unánime otra funcionalidad que no se incluye en el método de análisis pero que implica también la personalización del contenido: se trata de los servicios "a la carta" o "bajo demanda", que se podrían definir como la posibilidad de que el usuario vea cualquier programa a cualquier hora del día y sin tener que estar delante del televisor en el momento concreto de la emisión. Por tanto, constituye una herramienta radicalmente distinta y que implica un nivel de personalización menor que la adaptación de la página web.

La totalidad de cadenas analizadas sí que cumplen los otros dos indicadores que conforman este parámetro: la sindicación de contenidos y las alertas o boletines electrónicos. En el primer caso, la llamada sindicación o redifusión de contenidos (RSS) consiste en la "exportación" de las informaciones que se publican en la web para su consulta en otro contexto distinto, gracias a la utilización de unos protocolos estandarizados. Por su parte, los envíos de alertas y boletines buscan sin duda la fidelización de la audiencia a través de dispositivos como teléfonos móviles o tabletas. Pues bien, en este sentido, las cadenas televisivas españolas parecen haberse concienciado de la necesidad de implementar nuevas funcionalidades para poder llegar a todo tipo de dispositivos y, por ende, a más audiencias potenciales.

\subsection{Empleo de herramientas de la Web 2.0}

El paso de la web estática 1.0 a la web social 2.0 ha sido uno de las transformaciones clave en el devenir de Internet y las cadenas de televisión no podían permanecer ajenas a ella. En ese sentido, el concepto de compartir contenidos se traduce en la publicación de los mismos en plataformas como Facebook referenciando el contenido habitual. Dicho de otro modo, el usuario lanza una especie de recomendación a todos aquellos que tienen acceso a su espacio personal en la red social o plataforma correspondiente. Una opción muy tenida en cuenta por todas las cadenas analizadas que, no sólo incluyen esta opción en sus páginas web, sino que además suelen hacerlo de manera muy visible, normalmente justo debajo del titular de la noticia y antes del inicio del texto, mostrando los iconos de las redes sociales y plataformas correspondientes.

Por lo que respecta a los blogs, sorprende en cierto modo que ni lasexta.es ni canalsur.es cuenten con ellos. Cierto es que la tipología de los existentes en el resto de las cadenas es variable, y van desde los correspondientes a programas o espacios concretos hasta los de personajes expertos en alguna materia, pasando por los de carácter más personal protagonizados por periodistas, presentadores o locutores que colaboran con dicha cadena, pero, en todo caso, suelen ser herramientas que generan bastante participación en forma de comentarios, y de hecho varias de las cadenas los recogen en su portada, por lo que su no inclusión puede interpretarse negativamente desde el punto de vista de la participación y, lo que es más importante, de la implicación de la audiencia.

\subsection{Presencia del medio en plataformas de la Web 2.0}

Las redes sociales son herramientas que forman parte de la vida cotidiana de la mayoría de los internautas de todo el mundo, y los españoles no son una excepción, sino 
todo lo contrario, el último informe presentado por el Observatorio Nacional de las Telecomunicaciones y de la Sociedad de la Información (2011) situaba a España como el tercer país del mundo -y el primero de la Unión Europea- en uso de las redes sociales en Internet. Asimismo, según el informe, Facebook es la más usada, mientras que Youtube y Twitter también obtienen cifras altas. Las cadenas analizadas parecen tener en cuenta estos datos, pues su presencia en las redes sociales puede calificarse como desigual: masiva en las plataformas más populares, como las mencionadas, incluyendo enlaces e iconos en las portadas de sus sitios web en lugares muy visibles; pero escasa en las redes sociales de carácter más profesional y especializado, como Flickr o LinkedIn. Por otra parte, este criterio es común a las distintas cadenas con independencia de su ámbito de actuación ya que todas ellas obtienen unos resultados muy similares en este parámetro (Tabla 2).

Tabla 2: Presencia del medio en plataformas de la Web 2.0. Fuente: Elaboración propia

\begin{tabular}{|c|c|c|c|c|c|c|c|c|c|c|}
\hline Indicador & 5 & rtve & & $n=0$ & (b) & 涪 & 3 & & 4 & $c$ \\
\hline Youtube & $x$ & $x$ & $x$ & & $x$ & $x$ & $x$ & & $x$ & $x$ \\
\hline Flickr & & $x$ & & & & & & & & \\
\hline RR. SS. propias & & $x$ & & & & & & & & \\
\hline Linkediln & & $x$ & $x$ & & & & & & $x$ & \\
\hline Facebook & $x$ & $x$ & $\mathrm{x}$ & $x$ & $x$ & $x$ & $x$ & $x$ & $x$ & $x$ \\
\hline Twitter & $x$ & $x$ & $x$ & $x$ & $x$ & $x$ & $x$ & $x$ & $x$ & $x$ \\
\hline Vínculos & $x$ & $x$ & $x$ & $x$ & $x$ & $x$ & $x$ & $x$ & $x$ & $\mathrm{x}$ \\
\hline
\end{tabular}

Por último, el único caso de red social propia encontrado en el análisis es el de $L a$ Villa, comunidad creada por rtve.es y dirigida de manera específica a los aficionados al mundo del deporte. La participación exige registro del usuario y su presentación es bastante descriptiva: "La primera comunidad de deporte en España donde vivir el deporte con tus amigos y los deportistas. En La Villa el deporte eres tú"

Figura 2: Portada de La Villa. Fuente: rtve.es (05-02-2013)

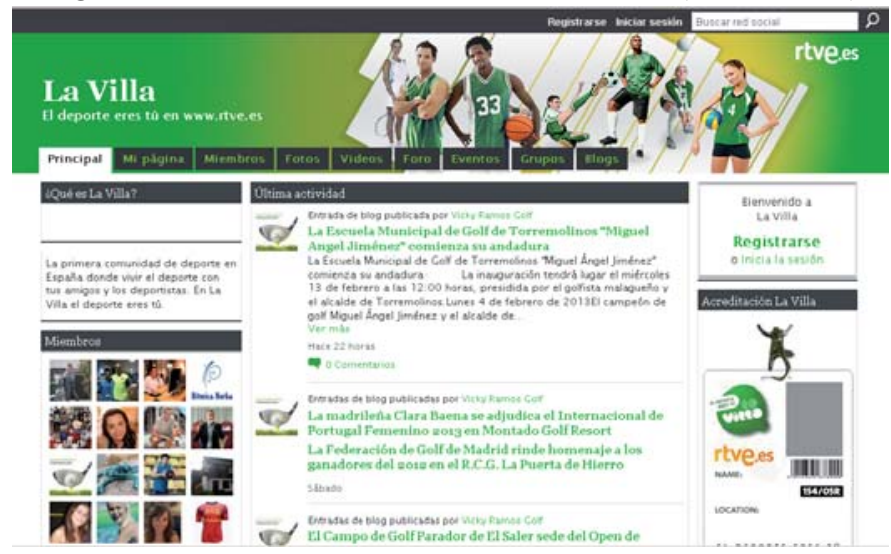




\section{Conclusiones}

El camino emprendido por las cadenas de televisión españolas durante los últimos años no ha supuesto ninguna excepción a la tendencia general que han seguido la mayoría de medios de comunicación en ese periodo, intentando adaptar las herramientas y características propias de Internet al contenido de sus sitios web y tratando de aumentar la interactividad y de fomentar la participación de las audiencias.

De acuerdo al análisis realizado, los aspectos o parámetros en los que se han dado pasos adelante mas evidentes han sido el empleo de las herramientas propias de la web 2.0, el acceso a la información, el registro del usuario y, en menor medida, la creación de canales de contenido personalizado y la presencia del medio de comunicación en plataformas de la web 2.0, como puede verse en el análisis global de resultados por cadenas y por parámetros (Tabla 3 ).

Por el contrario, la gran asignatura pendiente de los sitios web de las principales cadenas de televisión españolas sería la creación de contenidos por parte del usuario, es decir, la transformación del rol de usuario pasivo en usuario que incluso produce contenidos, convirtiendo el sitio web en un entorno de comunicación activa y permanente. En otras palabras, gracias a las transformaciones llevadas a cabo durante los últimos años, el usuario ya puede acceder, compartir y participar en el proceso de creación de contenidos, pero faltaría dar ese último paso consistente en asumir el papel protagonista en ese mismo proceso.

Por otro lado, los resultados de la adaptación de los sitios web no se traducen en estos momentos en diferencias muy significativas entre las cadenas públicas y las privadas, ni tampoco entre las distintas cadenas de ámbito estatal, excepción hecha de lasexta.es cuyo sitio web muestra, de acuerdo a los resultados del análisis, unos niveles de calidad menores al resto. Del mismo modo, esos niveles de calidad son ligeramente superiores entre las cadenas estatales que entre las autonómicas, exceptuando nuevamente a lasexta.es y, en este caso, también a telemadrid.es, que supera al resto de cadenas analizadas con independencia de su ámbito de actuación. Finalmente, dejando a un lado a la cadena pública madrileña, el resto de televisiones autonómicas obtienen unos resultados muy similares.

Tabla 3: Resultados totales. Fuente: elaboración propia

\begin{tabular}{|c|c|c|c|c|c|c|c|c|c|}
\hline & Interacc. & Contenidos & Registro & Acceso & Personal. & Web 2.0 & Platafs. & Total & $\%$ \\
\hline 5 & 3 & 0 & 2 & 6 & 2 & 2 & 4 & 19 & 59,37 \\
\hline rtve & 2 & 0 & 2 & 7 & 2 & 2 & 7 & 22 & 68,75 \\
\hline (3) & 3 & 0 & 2 & 7 & 2 & 2 & 5 & 21 & 65,62 \\
\hline (6) & 3 & 2 & 2 & 6 & 2 & 2 & 3 & 20 & 62,50 \\
\hline 滋 & 3 & 0 & 2 & 6 & 2 & 1 & 4 & 16 & 50,00 \\
\hline
\end{tabular}




\begin{tabular}{|c|c|c|c|c|c|c|c|c|c|}
\hline 3 & 3 & 0 & 2 & 5 & 2 & 2 & 4 & 18 & 56,25 \\
\hline ftry & 1 & 2 & 2 & 4 & 2 & 2 & 3 & 16 & 50,00 \\
\hline C & 4 & 3 & 1 & 7 & 2 & 2 & 5 & 24 & 75,00 \\
\hline Total & 26 & 7 & 16 & 60 & 20 & 18 & 43 & 190 & 59,37 \\
\hline$\%$ & 43,33 & 14,00 & 80,00 & 85,71 & 66,66 & 90,00 & 61,42 & 59,37 & \\
\hline
\end{tabular}

Por tanto, de cara al futuro, una de las posibles vías para mejorar la calidad de los sitios web pasaría por dotar al usuario de mayores oportunidades en la creación de los contenidos. También parece razonable, especialmente a tenor de los datos del estudio de la AIMC sobre televisión e Internet, poner en marcha medidas encaminadas a potenciar la multitarea (ver televisión y navegar al mismo tiempo).

En todo caso, será necesario estar atentos a las innovaciones que a buen seguro se irán produciendo ya que es posible que el camino hacia la convergencia tecnológica nunca llegue a estar plenamente y siempre se encontrará en transición por la sencilla razón de que esos avances son continuos y además se suceden a tal velocidad que la puesta al día es una tarea prácticamente inalcanzable.

\section{Referencias bibliográficas}

ASOCIACIÓN PARA LA INVESTIGACIÓN EN MEDIOS DE COMUNICACIÓN (AIMC) (2012): Radio: Tradicional vs Online, 2012, http://www.aimc.es/-La-Television-Tradicional-vs,193-.html [fecha de consulta: 12 de marzo de 2013]

ARJONA MARTÍN, José Borja (2009): "Los nuevos canales audiovisuales basados en web: RTVE.es". Revista Icono14, no 15, pp. 98-113, http://www. icono14.net/revista /num15/07_icono15_joseborjaarjona.pdf [fecha de consulta: 12 de marzo de 2013]

BOWMAN, Shayne y WILLIS, Chris (2003): We Media: How audiences are shaping the future of news and information. Reston, Va, The Media Center at the American Press Institute. Hypergene: http://www.hypergene.net/ [fecha de consulta: 10 de marzo de 2013]

CABRERA GONZÁLEZ, María Ángeles (2000): La prensa online. Los periódicos en la www. Barcelona, CIMS, 2000

CABRERA GONZÁLEZ, María Ángeles (2005): "Retos éticos del ciberperiodismo", en SALAVERRÍA, Ramón (coord.): Cibermedios. El impacto de Internet en los medios de comunicación. Sevilla, Comunicación Social, pp. 305-339.

CABRERA GONZÁLEZ, Ma Ángeles (2009): "La interactividad de las audiencias en entornos de convergencia digital". Revista Icono14, no 15, 2009, pp. 164-177, http://www. icono14.net/Num.-15.-Interactividad/la-interactividad-de-audiencias, [fecha de consulta: 10 de marzo de 2013] 
CEBRIÁN, Mariano (2004): Modelos de televisión: generalista, temática y convergente con Internet. Barcelona, Paidós.

DÍAZ NOCI, Javier et al (2009). "Content and message analysis of online journalism: some methodological proposals". Proceedings of the VInternational Conference: Comunication and Reality, pp. 647-656.

DÍAZ NOCI, Javier (2010): "Medios de comunicación en internet: algunas tendencias" en El profesional de la información, no 19, pp. 561-567.

GARCÍA DE TORRES, Elvira y POU, María José (2000): "Interactividad, información, promoción y valor de portal de las televisiones locales en la Red". Revista Latina de Comunicación Social, http://www.ull.es/publicaciones/latina /aa2000tma/132/Elvira.html, [fecha de consulta: 10 de marzo de 2013]

GARCÍA TORRE, Miguel (2012): “La nueva página web de la Compañía Radio Televisión de Galicia: una adaptación a los cambios de la red y a las demandas de los usuarios". Miguel Hernández Communication Journal, $\mathrm{n}^{\circ}$ 3, pp. 175-191: http://mhcj.es/2012/09/30/manuel_garcia/, [fecha de consulta: 10 de marzo de 2013]

GUALLAR, Javier y ABADAL, Ernest (2009): "Evaluación de las hemerotecas de prensa digital: indicadores y ejemplos de buenas prácticas". El profesional de la información, $\mathrm{n}^{\circ}$ 18, pp. 255-269.

LARA, Tíscar (2005): Hacia una televisión más participativa. Universidad Carlos III de Madrid, http://tiscar.com/2005/11/23/television-y-participacion, [fecha de consulta: 10 de marzo de 2013]

LERMA, Antonio (2011): "Convergencia de la televisión con Internet. Estudio de la televisión y su implantación en la Web”, II Congreso Internacional Comunicación 3.0: Nuevos Medios, Nueva Comunicación: http://comunicacion3punto0 .files.wordpress.com/2011/05/comunicacion3punto0libroactas2010.pdf [fecha de consulta: 10 de marzo de 2013]

LÓPEZ GARCÍA, Xosé (2011): "Rasgos de la estrategia de los cibermedios gallegos en su empeño por promover la experimentación para el cambio de modelo periodístico". Estudios sobre el Mensaje Periodístico, vol. 17, n 1, pp. 81-93. Madrid, Servicio de Publicaciones de la Universidad Complutense.

MASIP, Pere; DÍAZ-NOCI, Javier; DOMINGO, David; MICÓ-SANZ, Josep-Lluís; SALAVERRÍA, Ramón (2010): "Investigación internacional sobre ciberperiodismo: hipertexto, interactividad, multimedia y convergencia". El profesional de la información, $\mathrm{n}^{\circ} .19$, pp. 568-576.

MESO, Koldo; LARRONDO, Ainara (2011): Cambios en las estrategias de las audiencias de las páginas web de televisión. Análisis de la participación en los entes públicos autonómicos en España". II Congreso Internacional Comunicación 3.0: Nuevos Medios, Nueva Comunicación: http://comunicacion3punto0.files.wordpress.com/2011/05/comunicacion3punto0libroactas2010.pdf [fecha de consulta: 10 de marzo de 2013] 
MESO, Koldo; PALOMO, Bella; GANZABAL María (2009): “Análisis del periodismo participativo en las principales cadenas de televisión en la era de Internet. Los casos de antena 3.com, telecinco.es, plus.es, lasexta.com, cuatro.com y rtve.es", en LEÓN, Bienvenido: Telerrealidad. El mundo tras el cristal. Sevilla, Comunicación Social, pp. 192-204.

NAVARRO, Lizy (2009): “Tres lustros del periodismo digital: interactividad e hipertextualidad". Comunicar, $\mathrm{n}^{\circ} 33$, pp. 35-43.

OBSERVATORIO NACIONAL DE LAS TELECOMUNICACIONES Y DE LA SI (2011): "Estudio sobre el conocimiento y uso de las Redes Sociales en España": http://www.slideshare.net/retelur/redes-sociales-en-internet-ontsi-dic11, [fecha de consulta: 10 de marzo de 2013]

ODRIOZOLA, Javier (2012): “Análisis de contenido de los cibermedios generalistas españoles. Características y adscripción temática de las noticias principales de portada". Comunicación y Sociedad, vol. 25, nº 2, pp. 279-304.

ORIHUELA, José Luis (2002): Los 10 paradigmas de la e-comunicación, $\mathrm{http}: / /$ www.agetec.org /ageteca/Los\%2010Paradigmas\%20de $\% 201 \mathrm{a} \% 20 \mathrm{e}-\mathrm{Comu}-$ nicacion.pdf [fecha de consulta: 12 de febrero de 2013]

PALACIOS, Marcos y DÍAZ NOCI, Javier (eds., 2009): Online Journalism: Research Methods: A multidisciplinary approach in comparative perspective. Bilbao, Servicio Editorial de la Universidad del País Vasco.

PATERSON, Chris y DOMINGO, David (2008): Making online news: The etnography of new media production. New York, Peter Lang.

PÉREZ DE SILVA, Javier (2000): La televisión ha muerto. La nueva producción audiovisual en la era de Internet: La tercera revolución industrial. Barcelona, Gedisa.

PÉREZ DASILVA, Jesús A. y SANTOS DÍEZ (2009): "Las televisiones locales del País Vasco en Internet”. Revista Latina de Comunicación Social, n 64, pp. 192202.

RODRÍGUEZ-MARTÍNEZ, Ruth; CODINA, Lluis y PEDRAZA-JIMÉNEZ, Rafael (2010): "Cibermedios y web 2.0: modelo de análisis y resultado de aplicación". El profesional de la información, $\mathrm{n}^{\circ}$ 19, pp. 35-44.

RODRÍGUEZ-MARTÍNEZ, Ruth; CODINA, Lluis y PEDRAZA-JIMÉNEZ, Rafael (2012). "Indicadores para la evaluación de la calidad en cibermedios: análisis de la interacción y de la adopción de la Web 2.0". Revista Española de Documentación Científica, no 35, pp. 61-93.

SALAVERRÍA, Ramón; GARCÍA-AVILÉS, José A.; MASIP, Pere (2010): “Concepto de convergencia periodística”, en LÓPEZ-GARCÍA, Xosé y PEREIRA-FARIÑA, Xosé (coords.): Convergencia digital. Reconfiguración de los medios de comunicación en España. Santiago de Compostela, Servicio Editorial de la Universidad de Santiago de Compostela, pp. 41-63. 
Jesús Díaz-Campo L Las cadenas de televisión españolas en Internet: un estudio sobre la calidad...

TREMAYNE, Mark; WEISS, Amy Schmitz; \& ALVES, Rosental Calmon (2007): "From product to service: The diffusion of dynamic content in online newspapers". Journalism \& Mass Communication Quaterly, $\mathrm{n}^{\circ}$ 84, pp. 825-839.

ZAMITH, Fernando (2008): Ciberjornalismo: As potencialidades da Internet nos sites noticiosos portugueses. Porto, Afrontamento. 